\title{
ON SEMIPERFECT AND PERFECT RINGS
}

\author{
F. L. SANDOMIERSKI
}

Koh [2] has shown that a commutative ring with identity is semiperfect if and only if every simple right module has a projective cover. The purpose of this note is to show that the noncommutative version of Koh's characterization holds and to characterize right perfect rings as those for which every semisimple right module has a projective cover.

All rings considered have identity. A submodule $A$ of a right $R$ module $M, R$ a ring, is small in $M$ if $A+B=M$, for a submodule $B$ of $M$ implies $B=M$. The radical of a module $M_{R}$, denoted $J(M)$, is the intersection of all maximal submodules of $M$ if there are any and is $M$ otherwise, see e.g. [3].

The following proposition lists some useful and known results.

Proposition 1.

(a) $J(M)$ is the sum of all small submodules of a right $R$-module $M_{R}$.

(b) If $f \in \operatorname{Hom}\left(M_{R}, N_{R}\right)$ and $A_{R}$ is small in $M$, then $f\left(A_{R}\right)$ is small in $N$.

(c) $J\left(R_{R}\right)$ is the Jacobson radical of $R$.

(d) For a projective module $P_{R} \neq 0, J\left(P_{R}\right)=P J(R) \neq P$.

Proof. (a) [4, Hilssatz 2.1].

(b) $[4$, Hilfssatz 3.1] or [3, Example 8, p. 93].

(c) [3, Proposition 5, p. 58].

(d) [1, Remark p. 474].

LemMa 2. If $R$ is a ring and $J(R)=0$, then a module has a projective cover if and only if it is projective.

Proof. The "if" part is trivial. If $P \stackrel{\alpha}{\rightarrow} M \rightarrow 0$ is a projective cover of $M_{R}$, then $K=\operatorname{Ker}(\alpha)$ is small in $P$, so $K \subseteq J(P)=P J(R)=0$, so $\alpha$ is an isomorphism and the "only if" part follows.

Lemma 3. If $R$ is a ring with $J(R)=0$ and every simple right $R$ module has a projective cover, then $R$ is semisimple. (Here, semisimple means semisimple with minimum condition.)

Proof. The proof is essentially in the proof of [1, Lemma 2.5], but it will be given here for completeness.

Let $S$ be the right socle of $R$, i.e. $S$ is the sum of all simple right ideals. If $S \neq R$, then $S$ is contained in a maximal ideal $M$. By Lemma

Received by the editors March 15, 1968. 
2 and the hypothesis, $R / M$ is projective, so $M$ is a direct summand of $R$. Clearly, this contradicts that $S \subseteq M$, so $S=R$ and the lemma follows.

Theorem 4. A ring $R$ is semiperfect if and only if every simple right $R$-module has a projective cover.

Proof. Let $S$ be a simple right $R / J$ module, then $S$ is a simple right $R$-module, hence has an $R$-projective cover. By [1, Lemma 2.2], $S$ has an $R / J$ projective cover. Since $S$ was arbitrary, it follows by Lemma 3 that $R / J$ is semisimple. Let $R / I$ be a cyclic right $R$-module, where $I$ is a right ideal of $R$, then $R / I+J$ is a finitely generated right $R / J$ module, hence a direct sum of finitely many simple right $R$-modules. Since each simple right $R$-module has a projective cover, it follows by $[3$, Example 9, p. 93] that $R / I+J$ has a projective cover, say, $P \stackrel{\alpha}{\rightarrow} R / I+J \rightarrow 0$. The following diagram is commutative

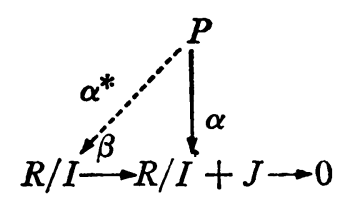

where $\beta$ is canonical and $\alpha^{*}$ exists since $P$ is projective. Since $K \operatorname{Rer}\left(\alpha^{*}\right)$ $\subseteq \operatorname{Ker}(\alpha)$, it follows that $\operatorname{Ker}\left(\alpha^{*}\right)$ is small in $P . \operatorname{Ker}(\beta)=I+J / I$ which is the image $J$ by the canonical map $R \rightarrow R / I$ so $I+J / I$ is small in $R / I$ by Proposition 1 (b) and the fact that $J$ is small in $R_{R}$ by Nakayama's Lemma [3, Example 14, p. 93]. Since $\alpha$ is onto and $\operatorname{Ker}(\beta)$ is small, it follows that $P \stackrel{\alpha^{*}}{\rightarrow} R / I \rightarrow 0$ is a projective cover of $R / I$, thus every cyclic right $R$-module has a projective cover, so $R$ is semiperfect by [1, Theorem 2.1]. The "only if" part follows from [1, Theorem 2.1].

THEOREM 5. A ring $R$ is right perfect if and only if every semisimple right $R$-module has a projective cover.

Proof. The "only if" part follows from [1, Theorem P].

For the "if" part it is sufficient to show that $J=J(R)$ is right $T$ nilpotent in view of Theorem 3 and $[1$, Theorem $\mathrm{P}]$. Let $F$ be a free right $R$-module; then since $R / J$ is semisimple, $F / F J$ is a semisimple right $R$-module, so has a projective cover $P \stackrel{\alpha}{\rightarrow} F / F J \rightarrow 0$. The following diagram is commutative:

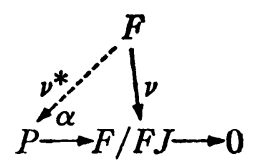


where $\nu$ is the canonical map and $\nu^{*}$ exists since $F_{R}$ is projective. Since $\nu$ is an epimorphism and $\operatorname{Ker}(\alpha)$ is small in $P$, it follows that $\nu^{*}$ is onto, so the exact sequence

$$
0 \rightarrow \operatorname{Ker}\left(\nu^{*}\right) \rightarrow F \rightarrow P \rightarrow 0
$$

splits since $P$ is projective, but then $K=\operatorname{Ker}\left(\nu^{*}\right) \subseteq F J$ is a direct summand of $F$. Clearly then $K=K \cap F J=K J$. Since $K$ is projective it follows that $K=0$ by Proposition $1(\mathrm{~d})$, so $\nu^{*}$ is an isomorphism and it follows that $F \stackrel{\nu}{\rightarrow} F / F J \rightarrow 0$ is a projective cover of $F$, so $F J$ is small in $F$.

Now let $F$ be a free right $R$-module with basis $\left\{x_{n} \mid n=1,2, \cdots\right\}$ and $\left\{a_{n} \mid n=1, \cdots\right\}$ a sequence in $J$. The submodule $B$ of $F$ generated by $\left\{x_{n}-x_{n+1} a_{n} \mid n=1,2, \cdots\right\}$ together with $F J$ generates $F$, so $F=B$ since $F J$ is small in $F$. In particular

$$
x_{1}=\sum_{i=1}^{n}\left(x_{i}-x_{i+1} a_{i}\right) r_{i}=r_{1} x_{1}+\sum_{i=2}^{n} x_{i}\left(r_{i}-a_{i-1} r_{i-1}\right)-x_{n+1} a_{n} r_{n} .
$$

By uniqueness of coefficients of the $x_{i}$ the coefficients of $x_{i}$ are zero for $i>1$ and $r_{1}=1$. But $r_{1}=1$ implies $r_{2}=a_{1}$, implies $r_{3}=a_{2} a_{1}$ and so on. $r_{n}=a_{n-1} \cdots a_{2} a_{1}$ and $a_{n} r_{n}=0$ so $J$ is right $T$-nilpotent.

\section{BIBLIOGRAPHY}

1. H. Bass, Finitistic dimension and a homological characterization of semi-primary rings, Trans. Amer. Math. Soc. 95 (1960), 466-488.

2. K. Koh, On a semi-primary ring, Proc. Amer Math. Soc. 19 (1968), 205-208.

3. J. Lambek, Lectures on rings and modules, Blaisdell, New York, 1966.

4. B. Pareigis, Radikale und Kleine Moduln, Bayer. Akad. Wiss. Math.-Natur. Kl. S.-B. 2 (1966), 185-199.

UNIVERSITY OF WISCONSIN 